\title{
Effects of Nutraceuticals and Botanicals on Macrophage Cholesterol Efflux: Implications for Atherosclerosis
}

\author{
Grace Megumi Sotherden, Harumi Uto-Kondo, Makoto Ayaori and Katsunori Ikewaki ${ }^{*}$
}

\author{
Division of Anti-Aging and Vascular Medicine, National Defense Medical College, Tokorozawa, Japan
}

\begin{abstract}
To date, the literature on high-density lipoprotein (HDL) levels as an inverse risk factor for atherosclerosis has mainly been observational, and it is likely that the metabolism and function of $\mathrm{HDL}$ is a more significant determinant of cardiovascular disease. As an example, as cholesterol is effluxed out of macrophages and carried to the liver via HDL for excretion, reduced cholesterol efflux can result in increased cholesterol accumulation. In terms of atherosclerosis risk, increasing cholesterol efflux is theoretically a strategy that can be considered as the groundwork of cardiovascular disease treatment and prevention. However, until now, there has not been a pharmaceutical agent that has effectively increased reverse cholesterol transport (RCT) at all steps of the process. Here is a review of the research on natural compounds present in edible foods and their observed in vitro and in vivo (and even ex vivo) effects on the first step of RCT: macrophage cholesterol efflux. The findings here are preliminary and contradictory, making it hard to translate the evidence on most of these naturally occurring agents into clinical applications.
\end{abstract}

Keywords: Nutrition, reverse cholesterol transport, HDL, CVD, lipoproteins.

\section{INTRODUCTION}

The Adult Treatment Panel III (ATPIII) cholesterol guidelines set LDL-cholesterol(C) as a primary target and first recommends therapeutic life style change (TLC), followed by drug therapy where HMG-CoA reductase inhibitors (statins) are mainly used to lower LDL-C. A recent meta-analaysis demonstrates that lowering LDL-C by statins reduces cardiovascular morbidity by about $30 \%$ [1]. At the same time, this fact also implies that there remains as much as $70 \%$ of risk which is independent of LDL-C lowering. Among the residual risk, high density lipoprotein ( $\mathrm{HDL}$ ) may play a pivotal role in reducing the risk since HDL exerts cardioprotective functions by promoting reverse cholesterol transport (RCT) via, for example, upregulation of key transporters, and thus efflux of cholesterol from macrophages lining arterial walls.

Macrophage cholesterol efflux to HDL is the first, and very critical, step in RCT from the periphery to the liver for excretion into bile or feces. Cholesterol efflux, in theory, decreases the likelihood of potentially deadly atheroma development in arteries. Impaired HDLmediated cholesterol efflux, on the other hand, is one of the factors that leads to cholesterol accumulation in macrophages. Therefore, increasing cholesterol efflux may be an effective strategy for atherosclerosis prevention and treatment.

Upregulation of the ATP-binding cassette subfamilies (ABCA1 and ABCG1) and the scavenger

*Address corresponding to this author at the Division of Anti-Aging and Vascular Medicine, National Defense Medical College, 3-2 Namiki, Tokorozawa, Saitama 359-8513, Japan; Tel: 81-4-2995-1211, ext. 2576, 2372; Fax: 81-4-2996-5202; E-mail: katsunorike@ndmc.ac.jp receptor class I, type $B(S R-B I)$, the most valuable players in RCT, have been shown to increase cholesterol efflux out of the macrophages that cluster in the arterial lining. SR-BI also mediates the selective uptake of cholesteryl esters to the hepatocytes, resulting in promoted RCT. Yet another player connected to cholesterol efflux is the lipogenic stearoyl CoA desaturase (SCD1) enzyme, which converts saturated fatty acids, i.e. palmitic acid, to monounsaturated fatty acids (MUFAs), i.e. palmitoleic acid. MUFAs tend to, more so than other fatty acids, be taken up into triacylglycerols and cholesteryl esters. In addition, PON1 and PON2 of the paraoxonases family have been found to be involved in protection against cholesterol-rich foam cell synthesis and atherogenesis. PON1 has been shown to be involved in HDL-mediated cholesterol efflux from macrophages. And finally, expression of $A B C A 1$ and $A B C G 1$ in macrophages is mediated by the nuclear receptor liver $X$ receptors $(L X R)$, which partner with retinoid $X$ receptors $(R X R)$ as heterodimers. Further, peroxisome proliferatoractivated receptors (PPAR) are thought to be involved in $A B C A 1$ expression upregulation.

Research in natural compounds have explored the concept of raising HDL-C, yet have yielded mixed results. For example, eicosapentaenoic acid (EPA) has been shown to increase HDL-C yet has decreased macrophage cholesterol efflux in vitro $[2,3]$. Rader et al. demonstrated the complicated nature of HDL with regard to metabolism and anti-atherosclerotic properties and proposed that the efflux hypothesis, instead of the HDL-C hypothesis, would relate to the cardioprotective properties attributed to HDL [4]. 


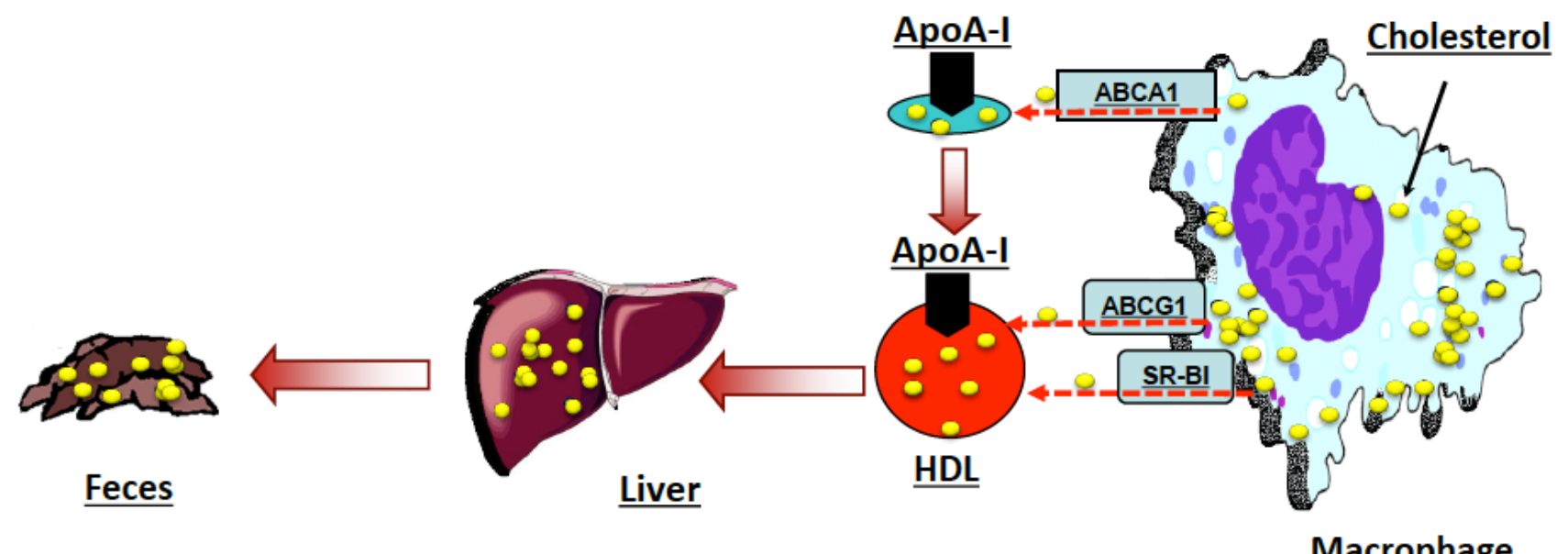

Figure 1: Reverse cholesterol transport in macrophages. Courtesy of Uto-Kondo, 2012.

The following paper is a comprehensive review of the existing literature on nutritional therapeutics and their effects on cholesterol efflux. A number of studies illuminate the usages of nutritional therapeutics for dyslipidemia and atherosclerosis, but some of the identified studies have not looked into the molecular mechanisms behind their anti-atherosclerotic properties with regard to cholesterol efflux. We narrowed the review to studies that found an effect of the intervention specifically at this first step of RCT, namely cholesterol efflux. To date there has not been, to the knowledge of the authors, a comprehensive review of the literature on nutraceuticals and macrophage cholesterol efflux.

The following terms (or combination of terms) were searched on PUBMED databases: atherosclerosis, fruits, vegetables, plants, botanicals, nutraceuticals, macrophage efflux, cholesterol efflux, food, nutrition, cholesterol flux, HDL metabolism, RCT, fatty acids, and food factors. All studies pertaining to cells other than macrophages were excluded. Studies that could not be found in full on PUBMED were excluded from the review.

\section{POLYPHENOLS, ANTIOXIDANTS AND OTHER NATURAL COMPOUNDS}

Resveratrol is a polyphenol found in red wine, berries, etc. Sevov et al. found that resveratrol increased LXR $\alpha$ mRA expression in both THP-1 derived macrophages and human macrophages [5]. $L X R \alpha$ regulates the expression of $A B C A 1, A B C G 1$, and apoE, which are key players in cholesterol efflux. Resveratrol caused apoE mRNA expression to increase threefold and ABCA1 mRNA expression to double in THP-1 macrophages, and ABCA1 and ABCG1 mRNA expression to increase in human macrophages. Berrougui, et al. found that resveratrol dose-dependently increased apoA-1-mediated cholesterol efflux. Based on the premise that oxidative damage to cells impairs cholesterol efflux, the authors induced oxidative stress on macrophages, and subsequently observed impaired HDL3-mediated cholesterol efflux, but resveratrol dose-dependently restored cholesterol efflux from these macrophages [6]. Voloshyna et al. found that resveratrol upregulated ABCA1, ABCG1, SR-BI and also 27-hydroxylase (which is involved in the excretion of intracellular cholesterol). At the same time, resveratrol suppressed foam cell formation in cholesterol-loaded macrophages [7].

In a recent study, curcumin, a component of the spice turmeric (Curcuma longa) was found to upregulate $A B C A 1$ expression, whereby increasing apoA-1-mediated cholesterol efflux from macrophages, with significant increases at 20 and $40 \mu \mathrm{M}$ compared to the control [8].

In a study by Xie et al., seven phenolic metabolites were identified from blueberry consumption: hippuric acid, 3-hydroxyphenylacetic acid, 3-hydroxybenzoic acid, ferulic acid, 3-(3-hydroxyphenyl) propionic acid, 3(4-hydroxyphenyl)propionic acid, and 3hydroxycinnamic acid [9]. The administration of these seven phenolic acids to Raw 264.7 macrophages in a separate study by the same authors, as compared to control macrophages, significantly increased (threefold) both the expression and protein levels of $A B C A 1$ $(P<0.05)[10]$.

Ingested anthocyanins are metabolized to various aromatic and phenolic compounds, such as the gut microbiotic metabolite protocatechuic acid (PCA) 
derived from cyanidin-3-O- $\beta$-glucoside (Cy-3-G), an anthocyanin pigment, and because absorption of anthocyanins has been proposed to be low, Wang et al. studied the effects of such a metabolite (PCA), previously shown to have anti-atherosclerotic properties, on RCT in apoE-knockout mice [11, 12]. The in vitro part of the study showed that PCA significantly increased macrophage cholesterol efflux to lipid-poor apo-A-1 or HDL; however, Cy-3-G was unable to show an effect on macrophage cholesterol efflux. In addition, ABCA1 and ABCG1 expression (but not SR-BI) increased in THP-1 macrophages. In mouse peritoneal macrophages (MPM) derived from mice fed PCA ( $5 \mathrm{mg} / \mathrm{kg}$ ) for 14 days during the in vivo part of the study, increased gene and protein expression of both ABCA1 and ABCG1 could be seen, in addition to increased cholesterol efflux to apoA-1 and HDL. Previously, Xia et al. had found that loading cells with these anthocyanin metabolites decreased macrophage and macrophage-derived foam cell intracellular cholesterol concentrations [13]. To confirm that this was not because of actions on cholesterol synthesis, the authors tested the $[3 \mathrm{H}]$ cholesterol efflux from the cells and found that it had increased 2-fold (compared to the control cells). This increase in cholesterol efflux was mediated by apoA-1. In a randomized, doubleblind, placebo-controlled study of dyslipidemic human subjects given twice-daily administration of $160 \mathrm{mg}$ of anthocyanin derived from berries for 12 weeks, HDLcholesterol concentrations were found to be increased (13.7\% in the anthocyanin group vs. $2.8 \%$ for placebo; $P<0.001$ ), and cholesterol efflux had increased (by $20.0 \%$ vs. $0.2 \%$ in the placebo group; $P<0.001$ ) [14].

Pomegranate juice's main polyphenols are anthocyanins, gallic and ellagic acids, ellagic tannins and catechins. Ellagic acid is also found in berries and some nuts. Park et al. found that ellagic acid increased macrophage cholesterol efflux by approximately 10 and $9 \%$ at $1 \mu \mathrm{mol} / \mathrm{L}$ and $5 \mu \mathrm{mol} / \mathrm{L}$, respectively, presumably due to the increase ABCA1 mRNA expression that was observed in this study (and most likely not due to SR-BI induction in this case) [15]. In Kaplan et al., pomegranate juice was given to apoE-deficient mice exhibiting advanced atherogenesis. Pomegranate juice significantly increased macrophage cholesterol efflux (by $39 \%$ ) as compared to the placebo [16]. Rosenblat et al. used macrophages from PON1- and PON2knockout mice given pomegranate juice, and found that, compared to the control macrophages, the treated macrophages exhibited a significant $22 \%$ increase in HDL-mediated cholesterol efflux [17]. The significance of this observation is that a defect of paraoxonase incurs a susceptibility to atherosclerosis, and according to this study, pomegranate juice would be able to ameliorate some aspects of this condition.

A study by Rosenblat et al. using polyphenol-rich beverages (mostly as pomegranate and black currant juices) demonstrated that in mice consuming these for at least one week, there was a significant decrease (of $8 \%$ ) in macrophage cholesterol accumulation, as compared to at baseline [18]. However, compared to at baseline, there was no significant effect on efflux of cholesterol from macrophages, indicating that some other mechanism was responsible for the decrease in cellular cholesterol content. Fuhrman et al. found that in macrophages that were incubated with pomegranate juice, no significant effect on cholesterol efflux from these cells were seen as compared to macrophages not treated with pomegranate juice [19]. However, the rate of cholesterol synthesis in the treated group macrophages was significantly decreased by $50 \%$ $(P<0.01)$ as compared to the control macrophages.

Uto-Kondo et al. had found that coffee, which is rich in the polyphenols caffeic acid and ferulic acid, increases cholesterol efflux from macrophages. In THP-1 macrophages, both caffeic and ferulic acids significantly increased HDL-mediated cholesterol efflux by $1.4+/-0.2$-fold $(p<0.01)$ and $1.4+/-0.3$-fold $(p<0.01)$, respectively, and upregulated the gene and protein expression of $A B C G 1$ and SR-BI, but not of $A B C A 1$. Given that $A B C A 1$ was not upregulated, it was not surprising that neither caffeic acid nor ferulic acid affected apoA-1-mediated cholesterol efflux. In addition, in human monocyte-derived macrophages (MDMs), caffeic acid increased HDL-mediated cholesterol efflux by $1.3+/-0.1$-fold $(p<0.01)$ and ferulic acid by $1.3+/-0.3$-fold $(p<0.05)$, with similar effects on the key transporters (as in THP-1 macrophages) and null effects on apoA-1-mediated cholesterol efflux. In an ex vivo human study, sera taken before and after coffee consumption was administered to corresponding human MDMs. In the MDMs cultured in sera derived after coffee consumption, the HDL-mediated cholesterol efflux increased by $1.4+/-0.6$-fold $(p<0.05)$ and $A B C G 1$ and $S R-B I$ protein expressions were upregulated by $1.7+/-0.4$-fold $(p<0.05)$ and $3.9+/-0.7-$ fold $(p<0.01)$, respectively, as compared to MDMs in the sera derived before consumption of coffee [20].

In a clinical, crossover, placebo-controlled study using human subjects [21], postmenopausal women were given isoflavone supplements vs. placebo. 
ABCA1-induced cholesterol efflux was not significantly different between the two groups. However, levels of pre- $\beta$ high-density lipoprotein (a subclass of highdensity lipoprotein) which is considered to have a greater efflux capacity, increased by $18 \%$ in the treatment group, while no other lipid parameters were affected.

A study on astaxanthin, a carotenoid found in salmon, lobster, crab and shrimp, showed that it upregulated $A B C A 1$ and $A B C G 1$ expression and apoA1- and HDL-mediated macrophage cholesterol efflux, particularly at higher concentrations of 50 and 100 $\mu \mathrm{mol} / \mathrm{L}[22]$.

Lycopene is a carotenoid found especially in tomatoes. Palozza et al. observed that ABCA1 protein expression increased by 2.2-fold in cells exposed to lycopene [23]. In addition, cav-1, a member of the caveolin family and associated with enhanced cholesterol efflux, was upregulated 2-fold in the treatment group. With these two findings, along with an observed intracellular cholesterol reduction, the authors deduced (without tracing of $[3 \mathrm{H}]$ cholesterol radioactivity) that cholesterol efflux had been enhanced. Then more recently, in 2012, researchers found lycopene to time-dependently increase mRNA and protein expression of PPARy, LXR- $\alpha$, and ABCA1, as well as change cholesterol concentrations both inside and outside the cell, all suggesting an induction of cellular cholesterol efflux [24].

Hesperetin, a citrus bioflavonoid, was found to upregulate $A B C A 1$ mRNA expression and significantly increased apoA-1-mediated cholesterol efflux from THP-1 macrophages at 5,10 , and $15 \mu \mathrm{M}$ of hesperetin $(p<0.05)[25]$.

Consumption of capsanthin, a primary carotenoid in paprika peppers (Capsicum annuum) resulted in a dose-dependent increase in HDL-cholesterol in rats $(r=0.597 ; p<0.005)$ [26]. Compared with rats fed a basal diet, the rats fed a basal diet with purified capsanthin added exhibited a $44 \%$ increase in mean plasma HDL-C $(p<0.05)$. In addition, both LCAT and apoA5 mRNA expressions were significantly upregulated, while apoA-1 mRNA expression was not significantly different. It is possible that capsanthin could increase cholesterol efflux.

Treatment of human THP-1 macrophages with Sallylcysteine (SAC), a compound found in garlic, at (10, 20 and $40 \mathrm{mM}$ for 24 hours) resulted in a dose- dependent increase in mRNA and protein expression of ABCA1 as compared to control, suggesting that SAC too could affect cholesterol efflux [27].

$\alpha$-Lipoic acid ( $\alpha$-LA), considered to be a free radical scavenger, increased the gene expression of ABCA1 and ABCG1 in $\alpha$-LA-treated J774.A1 macrophages (significant at 25 and $50 \mu \mathrm{M} ; \mathrm{p}<0.05$ ), but did not increase the expression of SR-BI or SR-A [28]. The mechanism behind the upregulation of $A B C A 1$ and ABCG1 was confirmed through the upregulation of LXR- $\alpha$. In human THP-1 macrophages, the same effects were seen. $\alpha$-LA significantly reduced lipid accumulation in cells and also significantly and dosedependently (at 12.5, 25 and $50 \mu \mathrm{M} ; \mathrm{p}<0.05$ ) increased cholesterol efflux from macrophages $(p<0.05)$.

Rosenblat et al. found that liposomal glutathione administration to apoE-deficient mice dosedependently increased HDL-induced macrophage cholesterol efflux by $78 \%$ (at $50 \mathrm{mg} / \mathrm{kg} /$ day) compared to the placebo-treated mice [29]. A seemingly contradictory finding was that consumption of liposomal glutathione by mice (at $50 \mathrm{mg} / \mathrm{kg} /$ day) lead to a significant decrease in HDL-C levels (by $21 \%$ ) as compared to in the control mice. Although increased cholesterol efflux from macrophages does not necessarily increase plasma HDL level, this discrepancy between cholesterol efflux and HDL-C in the plasma again points to the extent of the complexity of HDL's involvement in cholesterol homeostasis.

Rosa roxburghii (Chestnut Rose) was investigated by Zhang et al. using enzymatic fluorometric methods to quantify cellular cholesteryl ester (CE) accumulation in macrophages. They discovered that rosa roxburghii juice ameliorated the increase in CE accumulation (artificially induced by oxidized LDL) significantly at concentrations of $2 \mu \mathrm{l} / \mathrm{ml}$ (reduced by $55 \%$ ) and nearly completely reduced this increase (by $96 \%$ ) at $4 \mu \mathrm{l} / \mathrm{ml}$. In the latter part of the experiment, control cells incubated with HDL had their CE mass reduced by $64 \%$, demonstrating cholesterol efflux onto HDL, and rosa roxburghii juice also significantly reduced CE by $70 \%$ at $2 \mu \mathrm{l} / \mathrm{ml}$ and by $88 \%$ at $4 \mu \mathrm{l} / \mathrm{ml}$, also demonstrating cholesterol efflux mediated by rosa roxburghii juice [30].

Berrougui et al. looked at the effects of Marrubium vulgare (Horehound) extract on cholesterol efflux in human THP-1 macrophages. The extract dosedependently increased cholesterol efflux. In J774ABCA1-incubated macrophages, the authors did not 
observe an increase in HDL-mediated cholesterol efflux, indicating that the effects of $M$. vulgare increased cholesterol efflux in an ABCA1-independent manner [31]. The authors discussed that $M$. vulgare leaves contain high amounts of phenols. Whether or not its antioxidant properties are responsible for the increased HDL-mediated cholesterol efflux is not known.

Yoon et al. found that the lactic acid bacteria Lactobacillus rhamnosus BFE5264 and Lactobacillus plantarum NR74 induced LXR activation, thus increasing expression of $A B C A 1$ and $A B C G 1$, whereby also increasing cholesterol efflux in THP-1 macrophages [32].

In an ex vivo, randomized study by Sierksma et al. wherein the subjects were given $250 \mathrm{ml}$ of either white wine $(12 \%$ alcohol) or white grape juice, the white wine groups experienced an increase in serum HDL-C levels by $5 \%(p=0.02)$, as well as increased cholesterol efflux by $3.4 \%(p<0.05)$ [33]. The authors conclude that it is the alcoholic content of the wine that accounts for the difference in beneficial effects of the white wine over the white grape juice. However, a study considering the effects of $\mathrm{n}-3$ fatty acids and chronic alcohol consumption, found that while an $n-3$ fatty-acidenriched diet increased cholesterol efflux from macrophages by $79 \%$ compared to the control diet, an n-3 FA and alcohol-rich diet induced less of a beneficial effect (only of $25 \%$ compared to the control) [34]. Adding only alcohol to their diet decreased the cholesterol efflux from the macrophages by $21 \%$ $(p<0.01)$. Similarly, the same researchers studied moderate consumption of alcohol, simulated in mice. In macrophages derived from the mice fed alcohol as compared to from the control mice, the HDL-mediated cholesterol efflux was decreased by $20 \%(p<0.05)$ [35].

Niacin, a member of the vitamin B family, is used as a pharmaceutical agent in lowering lipids and in increasing HDL-C. In adipocytes treated with niacin, apoA-I-mediated cholesterol efflux was increased [36]. In addition, ABCA1 mRNA expression in these adipocytes was dose-dependently increased with exposure to a medium with varying amounts of niacin (0-1.0 mmol/L). In addition, given that ABCA1-mediated cholesterol efflux is transcriptionally regulated by PPARY and LXR $\alpha$, an assay demonstrated that niacin dose-dependently induced both PPARY and LXRa mRNA expression as well. Yvan-Charvet et al. compared niacin with anacetrapib (a cholesteryl ester transfer protein inhibitor) in a clinical trial to see which performed better in promoting HDL-mediated cholesterol efflux in macrophages [37]. Niacin, while it prompted a moderate increase in HDL-mediated cholesterol efflux (statistically significant at $25 \mathrm{ul}$ and 50 ul of the HDL-PEG supernatant), anacetrapib did so to a more significant degree. Clinical benefits of HDL-C raising therapy by niacin is yet to be proved.

\section{LIPIDS}

A study on phytosterols found that in THP-1 macrophages as well as in mouse peritoneal macrophages (MPMs), sitosterol had no effect on cholesterol efflux, while stigmasterol promoted efflux to apoA-1 by $25 \%(p<0.05)$ and also increased efflux to $H D L$, and increased expression of ABCA1 and ABCG1. Campesterol, on the other hand, modestly decreased efflux to HDL $(p<0.05)$ and did not affect the efflux to apoA-1 [38].

Fucosterol, derived from marine algae, in doses of either 100 or $200 \mu \mathrm{M}$, increased cholesterol efflux in HDL-treated THP-1-derived macrophages compared to control cells $(p<0.01)$ [39] (data not provided). The proposed mechanism is that it acts as an LXR-agonist.

Phosphatidylcholine, a major component of lecithin, was used in the form of soybean-derived phosphatidylcholine to incubate ECV304 human endothelial cells, resulting in a significant increase in cholesterol efflux (compared to controls) [40]. Lysophosphatidylcholine (LPC) dose-dependently (at $10,20,40,80 \mu \mathrm{M}$ ) increased expression of PPAR- $y$, LXR- $\alpha$, and ABCA1 in macrophage foam cells [41]. When these foam cells were incubated with $40 \mu \mathrm{M}$ of LPC for 12, 24 and 48 hours, they demonstrated timedependent increases in cholesterol efflux. In apoEdeficient mice, the cholesterol efflux seen by LPC treatment was significantly lower, implicating the importance of apoE. The authors, through the usage of specific inhibitors of PPARY, LXRa, and ABCA1, were able to confirm that LPC-induced cholesterol efflux from foam cells worked via a PPARY-LXRa-ABCA1dependent pathway, and was also mediated by apoE. In yet another study, in HDL taken from di-oleoylphosphatidylcholine-enriched serum, serum-mediated cholesterol efflux from J774 A.1 macrophages increased by $49 \%$ and HDL-mediated macrophage cholesterol efflux by $31 \%$ compared to control serum. In the in vivo part of the study, treatment mice were fed $0.2 \mathrm{ml}$ of olive oil every two days for 3 weeks, with placebo mice fed just water. HDL-mediated macrophage cholesterol efflux increased by $93 \%$ 
compared to in the placebo group. Next they extended the study to human subjects, having them consume $30 \mathrm{ml}$ of olive oil per day for 2 weeks. Compared to preconsumption of olive oil, serum-mediated macrophage efflux increased by $34 \%$ and HDL-mediated cholesterol efflux increased by $53 \%$ in the humans after olive oil consumption [42].

Extra virgin olive oil (EVOO), high in the MUFA oleic acid, and in antioxidants such as oleuropein, was looked at by Helal et al. in a human study in which the subjects consumed $25 \mathrm{ml} /$ day of EVOO for 12 weeks. In THP-1 macrophages, EVOO was able to increase cholesterol efflux by $9.8 \% \quad(p<0.01)$ [43]. EVOO increased cholesterol efflux from ABCA1-enriched J774 macrophages by $18.9 \% \quad(p<0.001)$ as compared to macrophages not enriched with ABCA1. In Fu5AH cells, SR-BI-mediated cholesterol efflux was increased by $14.8 \%(p<0.001)$ and HDL-mediated SR-BI-related cholesterol efflux was increased by $16.4 \%$ at 12 hours compared to baseline $(p<0.05)$. Also, in THP-1 macrophages, EVOO increased HDL-mediated cholesterol efflux by $11.9 \%(p<0.01)$ and upregulated ABCG1-induced HDL-mediated cholesterol efflux at 0 hours $(11.8 \%)$ and significantly more so at 12 hours (24.6\%), $\quad(p<0.001) . \quad$ In addition, HDL2-mediated cholesterol efflux in Fu5AH cells was increased by $24.1 \%$ at 12 hours compared to baseline $(p<0.05)$ while in ABCA1-enriched J774 macrophages, HDL3mediated cholesterol efflux was $15.2 \%$ higher than in control macrophages $(p<0.05)$. In human monocytederived macrophages (HMDMs), cholesterol efflux was increased by $44 \%(p<0.001)$. In the HMDMs, regulation of both gene and protein expression was increased in ABCA1 (27.51\%, $p<0.0001 ; 16.08 \%, \quad p<0.001$, respectively) and ABCG1 (26.48\%, $p<0.001 ; 35.79 \%$, $\mathrm{p}<0.01$, respectively). However, both gene and protein expression were downregulated in SR-BI (by 30\%, $\mathrm{p}<0.0001 ; 2.51 \%, \mathrm{p}<0.05$, respectively). Meanwhile, a study by Stein et al. on oleic acid, a monounsaturated fatty acid, found an inhibitory effect of macrophage cholesterol efflux to apoA-1 by oleic acid (by $27 \%$ ) as compared to the control cells [44].

Extra virgin olive oil enriched with green tea polyphenols (EVOO-GTPP) was compared to extra virgin olive oil alone (EVOO) in administration to apo-Edeficient mice exhibiting atherosclerosis [45]. EVOOGTPP contains MUFAs, primarily which is oleic acid, in addition to phytosterols, polyphenols, and tocopherols. Green tea contains polyphenols called catechins, the main one being epigallocatechin gallate (EGCG). EVOO compared to placebo significantly increased
HDL-mediated cholesterol efflux $(p<0.01)$ and EVOOGTPP, as compared to EVOO, even more drastically increased cholesterol efflux (the difference between EVOO-GTPP and EVOO being significant at $p<0.05$ ). Both had measureable effects on both apoA-1mediated and HDL-mediated cholesterol efflux. EVOO and EVOO-GTPP increased HDL-mediated cholesterol efflux by $42 \%$ and $139 \%$, respectively. In addition to the other parameters measured in this study (such as lesion size), the authors concluded that EVOO enriched with GTPPs can mitigate atherosclerosis in the apoE-deficient mice model.

Berrougui et al. studied the antioxidant component of argan oil, (from the seeds of Argania spinosa) particularly virgin argan oil phenolic extracts (VAO-PE), and found that incubation with $320 \mu \mathrm{g} / \mathrm{ml}$ VAO-PE increased cholesterol efflux from human THP-1 macrophages $(p=0.0245)$ [46]. In addition, when the experimenters induced oxidation of HDL with copper, cholesterol efflux from the THP-1 macrophages was decreased, and then reversed time-dependently by 320 $\mathrm{ug} / \mathrm{ml}$ of VAO-PE. Then in 2007, Berrougui et al. examined a methanolic extract of Argania spinosa L. pericarp (MEAP), which increased ABCA1-independent HDL-mediated cholesterol efflux in $\mathbf{J 7 7 4}$ macrophages [47].

One study tested various dietary fatty acids on macrophage cholesteryl ester accumulation and cholesterol efflux [48]. The diets of African green monkeys were supplemented with various fatty acids ( $n-3$, saturated, monounsaturated, and n-6 fatty acids). THP-1 cells incubated with the isolated lipids from each of these various treatment groups initially did not yield differences in cholesterol efflux. However, after the cells underwent triglyceride removal, n-3 fatty acids significantly and time-dependently increased cholesterol efflux compared the other fatty acids. Similarly, in 1990, Pal \& Davis had enriched cell lines with either n-6 PUFAs or n-3 PUFAs. N-3 PUFAs doubly increased the rate of cholesterol efflux from cells as did n-6 PUFAs [49].

However, Kralova Lesna et al. conducted a study in which replacing saturated fatty acids with PUFAs did not result in significant changes in cholesterol efflux [50]. In addition, in 2007, Buonacorso et al. did not find differences in cholesterol efflux in cells incubated in the plasma of subjects consuming diets of differing fatty acid composition (trans fatty acids vs. PUFAs vs. SFAs) [51]. However, the authors stated that it may be due to the low proportions of fatty acids ingested in the 
Table 1: Shows a Summary of the Results

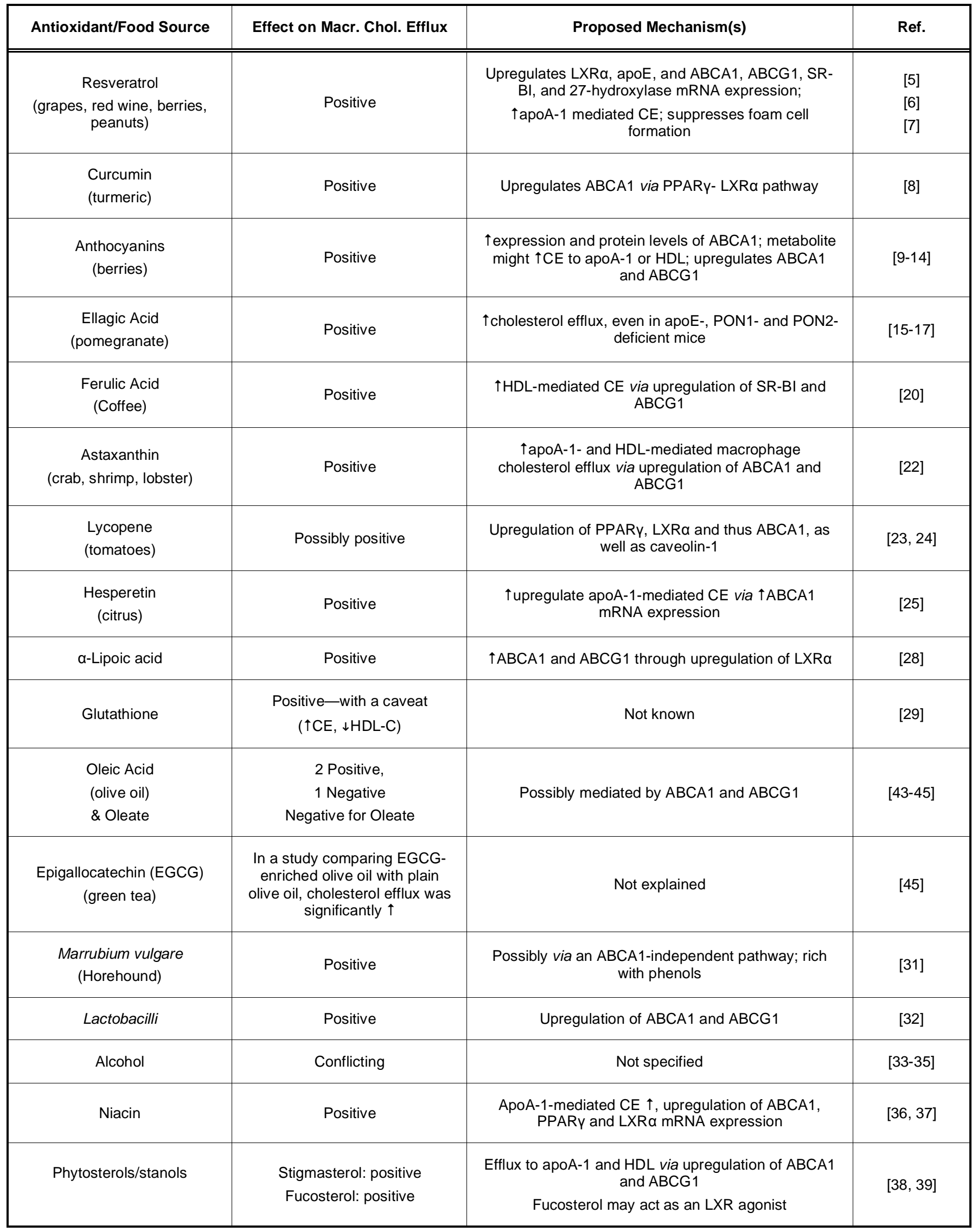


(Table 1). Continued.

\begin{tabular}{|c|c|c|c|}
\hline Antioxidant/Food Source & Effect on Macr. Chol. Efflux & Proposed Mechanism(s) & Ref. \\
\hline $\begin{array}{l}\text { Lysophosphatidylcholine } \\
\text { (LPC) }\end{array}$ & Positive & $\begin{array}{l}\text { PPARY- LXRa-ABCA1- and apoE-dependent } \\
\text { pathways; possibly through PON1 activation }\end{array}$ & {$[40-42]$} \\
\hline Argania Spinosa & Positive & ABCA1-independent & {$[46,47]$} \\
\hline Unsaturated Fatty Acids & $\begin{array}{l}\text { Conflicting; differences } \\
\text { between mono- and poly-UFAs }\end{array}$ & $\begin{array}{l}\text { Not specified; decreased effects might be due to } \\
\text { suppression of } A B C A 1 \text { and possibly of } A B C G 1 \text { as } \\
\text { well }\end{array}$ & $\begin{array}{l}{[48-50,52,} \\
53,58]\end{array}$ \\
\hline Saturated Fatty Acids & Conflicting & Stearate $\uparrow A B C A 1$ and $A B C G 1$ & {$[2,3,53]$} \\
\hline EPA \& DHA & Negative & $\begin{array}{c}\text { Inhibited ABCA } 1 \text { and possibly LCAT; PPAR } \uparrow \text { by } \\
\text { DHA; LXR } \downarrow \text { by EPA and DHA }\end{array}$ & [3] \\
\hline Linoleic Acid & Positive & $\begin{array}{c}\text { apoA-1-mediated } A B C A 1, A B C G 1 \text { and } S R-B I \\
\text { upregulation }\end{array}$ & {$[55,57]$} \\
\hline Walnut oil & Positive & FXR-mediated downregulation of SCD1 & {$[56]$} \\
\hline
\end{tabular}

Key: CE, cholesterol efflux; $\uparrow$, increased; $\downarrow$, decreased.

study, and the general similar composition of fatty acids between the three diet groups that no real big differences in cholesterol efflux between the groups were observed.

Uehara et al. studied the effects of the unsaturated fatty acids EPA, linoleic acid (LA), arachidonic acid (AraA) and saturated fatty acids palmitic acid (PA) and stearic acid (SA) on cholesterol transport [2]. What they found was that unsaturated fatty acids dosedependently suppressed ABCA1 gene expression and also reduced apoA-1-mediated cholesterol efflux in macrophages, LA exerting the strongest effects, then AraA, then EPA, then linoleic acid. PA, a saturated fatty acid, moderately suppressed ABCA1 while stearic acid moderately yet significantly increased ABCA1. Then in 2007 the same authors conducted another study in which the effects of unsaturated fatty acids and saturated fatty acids on cholesterol efflux were examined once more [3]. This time they found that ABCG1 gene expression was downregulated by these fatty acids. Meanwhile, saturated fatty acids transcriptionally upregulated promoters $A$ and $B$ of the ABCG1 gene. Stearic acid was found to siginifcantly increase promoters $A$ and $B$ activity, while palmitic acid only did for promoter B. ABCG1 gene expression was decreased by $48.6 \%$ by EPA and $49.6 \%$ by LA. Likewise, ABCG1 protein expression was decreased by $40.5 \%$ by EPA, $45.8 \%$ by LA, and $35.6 \%$ by OA. Ku et al. incubated macrophages with $100 \mathrm{umol} / \mathrm{L}$ of PA, palmitoleic acid, oleic acid, linolenic acid or EPA. Without an LXR agonist, ABCA1 and ABCG1 mRNA expression were significantly decreased with unsaturated fatty acid incubation as compared with the control; however, in the presence of an LXR agonist, $A B C A 1$ and $A B C G 1$ mRNA levels went back to normal. However, when the LXR agonist was present, the ABC transporter protein expressions were reduced [52]. Wang \& Oram found that incubation of macrophages with the unsaturated fatty acids oleate and linoleate greatly reduced apoA-1-mediated cholesterol efflux from macrophages (i.e. by more than $50 \%$ at 20 hours of incubation) in a dose-dependent manner, by degrading ABCA1 [53]. (Linoleate did more so than oleate.) Oleate maximally inhibited cholesterol efflux at 16 hours, whereas linoleate reached this in only 4 hours. In addition, unsaturated fatty acids decreased membrane ABCA1. Linoleate and oleate decreased ABCA1 in a dose-dependent manner. At the same time, saturated fatty acids did not have an effect on ABCA1, nor on apoA-1 binding to ABCA1. In particular, stearate, a saturated fatty acid, did not have an effect on apoA-1 binding to $A B C A 1$. The effect of the unsaturated fatty acids on $A B C A 1$ was not at the transcriptional level, as ABCA1 mRNA was not affected. What was discovered by the authors (and mentioned earlier in the paragraph), was that, instead ABCA1 degradation was increased by unsaturated fatty acids, and cholesterol efflux was inhibited.

$\mathrm{Hu}$ et al. demonstrated that EPA dose- and timedependently downregulated $A B C A 1$ protein expression (but not ABCA1 mRNA expression) and inhibited ABCA1-mediated cholesterol efflux particularly at the 
highest doses of EPA used in the study $(10 \mu \mathrm{M}$ and $100 \mu \mathrm{M})[54]$.

Kämmerer et al. observed that with 13-hydroxy linoleic acid (which is linoleic acid treated with an enzyme), apoA-1-mediated cholesterol efflux in macrophages was increased, as was the expression of $A B C A 1, A B C G 1$, and SR-BI [55].

Zhang et al. had found that, in foam cells derived from THP-1 macrophages treated with sera from human subjects fed walnut oil, this intervention significantly promoted cholesterol efflux by down regulating the expression of SCD1 [56]. The primary n3 PUFA in walnuts is a-linolenic acid, which was found to activate the nuclear receptor farnesoid-X-receptor (FXR) which was responsible for the reduction in SCD1 activity. Zhang et al. further conducted a study specifically using a-linolenic acid itself and had confirmed that this reduction in SCD1 expression was indeed mediated by FXR. The n-3 PUFA a-linolenic acid had, in this study as well, increased cholesterol efflux in macrophage-derived foam cells via the inhibition of SCD1 [57].

\section{CONCLUSION}

Regarding lipids, the inconsistencies of the findings on cholesterol transport make them very hard to interpret. For example, regarding unsaturated fatty acids, Uehara et al. and Wang \& Oram and $\mathrm{Ku}$ et al. had found an inhibitory effect of UFAs on cholesterol efflux [2, 3, 52, 53], while Kämmerer et al., Zhang et al., Helal et al. found stimulatory actions on unsaturated fatty acids of varying numbers of double bonds (monovs. poly) on cholesterol efflux $[43,55,57]$.

As atherosclerotic disease is the basis for many morbidities, such as peripheral vascular disease, myocardial infarction, and ischemic stroke, the development of therapeutic interventions targeting atherosclerosis and its risk factors are in high demand. These are preliminary studies that describe cellular mechanisms of the effects of nutraceutical and botanical substances on HDL functionality. However, one cannot yet make inferences about how, at the studied doses, these therapeutics would act in the human body in terms of macrophage cholesterol efflux. Also, it is of value to note some contradictions in the literature. The effects of MUFAs tend to be unfavorable in that they decrease cholesterol efflux in macrophages, thus increasing the retention of intracellular cholesterol. This is inconsistent with the favorable effects that MUFAs in the diet have on lipid and lipoprotein profiles, namely reduction of LDL, in clinical trials. At the same time, there are also many conflicting results (some positive, some negative) regarding MUFAs and atherosclerosis in animal studies. This is a gap in the research that necessitates further research.

During the last decade starting with the cornerstone discovery of ABCA1, novel small molecules promoting antiatherogenic properties of HDL have yet to be identified. In this regard, the results of these in vitro and in vivo studies are indeed provocative. The current body of evidence presented here agrees with other studies on the cardioprotective, hypolipidemic, antioxidative and anti-inflammatory properties of these natural compounds, strengthening the argument that there may be some potential here for the usage of these natural compounds in atherosclerosis prevention and treatment. This is yet another reason to continue investigation in this field.

\section{ABBREVIATIONS AND ACRONYMS}

\begin{tabular}{|c|c|c|}
\hline $\mathrm{ABCA} 1$ & & $\begin{array}{l}\text { ATP-binding cassette subfamily A member } \\
1\end{array}$ \\
\hline ABCG1 & & $\begin{array}{l}\text { ATP-binding cassette subfamily } G \text { member } \\
1\end{array}$ \\
\hline Apo & $=$ & apolipoprotein \\
\hline CVD & $=$ & cardiovascular disease \\
\hline HDL & $=$ & high-density lipoprotein \\
\hline LDL & $=$ & low-density lipoprotein \\
\hline LXR & $=$ & liver $\mathrm{X}$ receptor \\
\hline MDM & $=$ & human monocyte-derived macrophages \\
\hline MPM & $=$ & mouse peritoneal macrophages \\
\hline PON & $=$ & paraoxonases \\
\hline PPAR & $=$ & peroxisome proliferator-activated receptor \\
\hline $\mathrm{RCT}$ & $=$ & reverse cholesterol transport \\
\hline SCD & $=$ & stearoyl CoA desaturase \\
\hline $\mathrm{R}-\mathrm{BI}$ & $=$ & scavenger receptor class B type I \\
\hline
\end{tabular}

\section{REFERENCES}

[1] Baigent $C$, et al. Efficacy and safety of more intensive lowering of LDL cholesterol: a meta-analysis of data from 
170,000 participants in 26 randomised trials. Lancet 376(9753): 1670-81.

[2]

Uehara $\mathrm{Y}$, et al. Polyunsaturated fatty acids and acetoacetate downregulate the expression of the ATPbinding cassette transporter A1. Diabetes 2002; 51(10): 2922-8.

http://dx.doi.org/10.2337/diabetes.51.10.2922

Uehara $\mathrm{Y}$, et al. Unsaturated fatty acids suppress the expression of the ATP-binding cassette transporter G1 (ABCG1) and ABCA1 genes via an LXR/RXR responsive element. Atherosclerosis 2007; 191(1): 11-21.

http://dx.doi.org/10.1016/j.atherosclerosis.2006.04.018

[4] Rader DJ, et al. The role of reverse cholesterol transport in animals and humans and relationship to atherosclerosis. $J$ Lipid Res 2009; 50(Suppl): S189-94. http://dx.doi.org/10.1194/jlr.R800088-JLR200

[5] Sevov M, Elfineh L, Cavelier LB, Resveratrol regulates the expression of LXR-alpha in human macrophages. Biochem Biophys Res Commun 2006; 348(3): 1047-54. http://dx.doi.org/10.1016/..bbrc.2006.07.155

[6] Berrougui $\mathrm{H}$, et al. A new insight into resveratrol as an atheroprotective compound: inhibition of lipid peroxidation and enhancement of cholesterol efflux. Atherosclerosis 2009; 207(2): 420-7.

http://dx.doi.org/10.1016/i.atherosclerosis.2009.05.017

[7] Voloshyna I, et al. Resveratrol mediates anti-atherogenic effects on cholesterol flux in human macrophages and endothelium via PPARgamma and adenosine. Eur $\mathrm{J}$ Pharmacol

[8] Zhao JF, et al. Molecular mechanism of curcumin on the suppression of cholesterol accumulation in macrophage foam cells and atherosclerosis. Mol Nutr Food Res 56(5): 691-701.

[9] Xie $\mathrm{C}$, et al. Blueberries reduce pro-inflammatory cytokine TNF-alpha and IL-6 production in mouse macrophages by inhibiting NF-kappaB activation and the MAPK pathway. Mol Nutr Food Res 55(10): 1587-91. http://dx.doi.org/10.1002/mnfr.201100344

[10] Xie C, et al. Phenolic acids are in vivo atheroprotective compounds appearing in the serum of rats after blueberry consumption. J Agric Food Chem 59(18): 10381-7. http://dx.doi.org/10.1021/if2025264

[11] Wang D, et al. Protocatechuic acid, a metabolite of anthocyanins, inhibits monocyte adhesion and reduces atherosclerosis in apolipoprotein E-deficient mice. J Agric Food Chem 58(24): 12722-8.

[12] Wang D, et al. Gut Microbiota Metabolism of Anthocyanin Promotes Reverse Cholesterol Transport in Mice Via Repressing miRNA-10b. Circ Res 111(8): 967-81.

[13] Xia $M$, et al. Anthocyanins induce cholesterol efflux from mouse peritoneal macrophages: the role of the peroxisome proliferator-activated receptor \{gamma\}-liver $X$ receptor \{alpha\}-ABCA1 pathway. J Biol Chem 2005; 280(44): 36792801.

[14] Qin Y, et al. Anthocyanin supplementation improves serum LDL- and HDL-cholesterol concentrations associated with the inhibition of cholesteryl ester transfer protein in dyslipidemic subjects. Am J Clin Nutr 2009; 90(3): 485-92.

[15] Park SH, et al. Dietary ellagic acid attenuates oxidized LDL uptake and stimulates cholesterol efflux in murine macrophages. J Nutr 141(11): 1931-7.

[16] Kaplan $M$, et al. Pomegranate juice supplementation to atherosclerotic mice reduces macrophage lipid peroxidation, cellular cholesterol accumulation and development of atherosclerosis. J Nutr 2001; 131(8): 2082-9.

[17] Rosenblat M. Volkova N, Aviram M. Pomegranate juice (PJ) consumption antioxidative properties on mouse macrophages, but not PJ beneficial effects on macrophage cholesterol and triglyceride metabolism, are mediated via PJ- induced stimulation of macrophage PON2. Atherosclerosis 212(1): 86-92.

[18] Rosenblat $M$, et al. Consumption of polyphenolic-rich beverages (mostly pomegranate and black currant juices) by healthy subjects for a short term increased serum antioxidant status, and the serum's ability to attenuate macrophage cholesterol accumulation. Food Funct 1(1): 99-109.

[19] Fuhrman B, Volkova N, Aviram M. Pomegranate juice inhibits oxidized LDL uptake and cholesterol biosynthesis in macrophages. J Nutr Biochem 2005 16(9): 570-6.

[20] Uto-Kondo $\mathrm{H}$, et al. Coffee consumption enhances highdensity lipoprotein-mediated cholesterol efflux in macrophages. Circ Res 106(4): 779-87.

[21] Badeau $R$, et al. Effect of isolated isoflavone supplementation on ABCA1-dependent cholesterol efflux potential in postmenopausal women. Menopause 2007; 14(2): 293-9.

[22] lizuka $M$, et al. Astaxanthin enhances ATP-binding cassette transporter A1/G1 expressions and cholesterol efflux from macrophages. J Nutr Sci Vitaminol (Tokyo) 58(2): 96-104.

[23] Palozza $\mathrm{P}$, et al. Lycopene regulation of cholesterol synthesis and efflux in human macrophages. J Nutr Biochem 22(10): 971-8.

[24] Yang CM, et al. Lycopene and the LXRalpha agonist T0901317 synergistically inhibit the proliferation of androgenindependent prostate cancer cells via the PPARgammaLXRalpha-ABCA1 pathway. J Nutr Biochem 23(9): 1155-62.

[25] lio $A$, et al. Hesperetin upregulates $A B C A 1$ expression and promotes cholesterol efflux from THP-1 macrophages. J Nat Prod 75(4): 563-6.

[26] Aizawa K, Inakuma T. Dietary capsanthin, the main carotenoid in paprika (Capsicum annuum), alters plasma high-density lipoprotein-cholesterol levels and hepatic gene expression in rats. Br J Nutr 2009; 102(12): 1760-6.

[27] Malekpour-Dehkordi Z, et al. S-Allylcysteine, a Garlic Compound, Increases ABCA1 Expression in Human THP-1 Macrophages. Phytother Res.

[28] Cheng LC, et al. alpha-Lipoic acid ameliorates foam cell formation via liver $X$ receptor alpha-dependent upregulation of ATP-binding cassette transporters $A 1$ and $\mathrm{G} 1$. Free Radic Biol Med 50(1): 47-54

[29] Rosenblat $M$, et al. Anti-oxidant and anti-atherogenic properties of liposomal glutathione: studies in vitro, and in the atherosclerotic apolipoprotein E-deficient mice. Atherosclerosis 2007; 195(2): e61-8.

[30] Zhang $\mathrm{C}$, et al. Inhibitory effects of rosa roxburghii tratt juice on in vitro oxidative modification of low density lipoprotein and on the macrophage growth and cellular cholesteryl ester accumulation induced by oxidized low density lipoprotein. Clin Chim Acta 2001; 313(1-2): 37-43.

[31] Berrougui $\mathrm{H}$, et al. Marrubium vulgare extract inhibits humanLDL oxidation and enhances HDL-mediated cholesterol efflux in THP-1 macrophage. Life Sci 2006; 80(2): 105-12.

[32] Yoon HS, et al. The probiotic Lactobacillus rhamnosus BFE5264 and Lactobacillus plantarum NR74 promote cholesterol efflux and suppress inflammation in THP-1 cells. J Sci Food Agric.

[33] Sierksma A, et al. Effect of moderate alcohol consumption on parameters of reverse cholesterol transport in postmenopausal women. Alcohol Clin Exp Res 2004; 28(4): 662-6.

[34] Marmillot $P$, et al. Effect of dietary omega-3 fatty acids and chronic ethanol consumption on reverse cholesterol transport in rats. Metabolism 2000; 49(4): 508-12.

[35] Marmillot $\mathrm{P}$, et al. Long-term ethanol consumption impairs reverse cholesterol transport function of high-density lipoproteins by depleting high-density lipoprotein 
sphingomyelin both in rats and in humans. Metabolism 2007; 56(7): 947-53.

[36] Wu ZH, Zhao SP, Niacin promotes cholesterol efflux through stimulation of the PPARgamma-LXRalpha-ABCA1 pathway in 3T3-L1 adipocytes. Pharmacology 2009; 84(5): 282-7.

[37] Yvan-Charvet L, et al. Cholesterol efflux potential and antiinflammatory properties of high-density lipoprotein after treatment with niacin or anacetrapib. Arterioscler Thromb Vasc Biol 30(7): 1430-8.

[38] Sabeva NS, et al. Phytosterols differentially influence ABC transporter expression, cholesterol efflux and inflammatory cytokine secretion in macrophage foam cells. J Nutr Biochem 22(8): 777-83.

[39] Hoang MH, et al. Fucosterol Is a Selective Liver X Receptor Modulator That Regulates the Expression of Key Genes in Cholesterol Homeostasis in Macrophages, Hepatocytes, and Intestinal Cells. J Agric Food Chem.

[40] Mastellone I, et al. Dietary soybean phosphatidylcholines lower lipidemia: mechanisms at the levels of intestine, endothelial cell, and hepato-biliary axis. J Nutr Biochem 2000; 11(9): 461-6.

[41] Hou M, et al. Lysophosphatidylcholine promotes cholesterol efflux from mouse macrophage foam cells via PPARgammaLXRalpha-ABCA1-dependent pathway associated with apoE. Cell Biochem Funct 2007; 25(1): 33-44.

[42] Efrat $\mathrm{M}$, et al. Di-oleoyl phosphatidylcholine (PC-18:1) stimulates paraoxonase 1 (PON1) enzymatic and biological activities: in vitro and in vivo studies. Atherosclerosis 2009; 202(2): 461-9.

[43] Helal O, et al. Extra-virgin olive oil consumption improves the capacity of HDL to mediate cholesterol efflux and increases ABCA1 and ABCG1 expression in human macrophages. $\mathrm{Br} \mathrm{J}$ Nutr 1-12.

[44] Stein $O$, et al. Effects of oleic acid and macrophage recruitment on cholesterol efflux in cell culture and in vivo. Nutr Metab Cardiovasc Dis 2008; 18(9): 596-601.

[45] Rosenblat $M$, et al. Antiatherogenicity of extra virgin olive oil and its enrichment with green tea polyphenols in the atherosclerotic apolipoprotein-E-deficient mice: enhanced macrophage cholesterol efflux. J Nutr Biochem 2008; 19(8): 514-23.

[46] Berrougui $\mathrm{H}$, et al. Phenolic-extract from argan oil (Argania spinosa L.) inhibits human low-density lipoprotein (LDL) oxidation and enhances cholesterol efflux from human THP-1 macrophages. Atherosclerosis 2006; 184(2): 389-96.
[47] Berrougui $\mathrm{H}$, et al. Antiatherogenic activity of extracts of Argania spinosa L. pericarp: beneficial effects on lipid peroxidation and cholesterol homeostasis. Can J Physiol Pharmacol 2007; 85(9): 918-27.

[48] Lada AT, Rudel LL, St Clair RW. Effects of LDL enriched with different dietary fatty acids on cholesteryl ester accumulation and turnover in THP-1 macrophages. J Lipid Res 2003; 44(4): 770-9.

[49] Pal S, Davis PJ, N-3 polyunsaturated fatty acids enhance cholesterol efflux from human fibroblasts in culture. Biochem Biophys Res Commun 1990; 173(2): 566-70.

[50] Kralova Lesna I, et al. Replacement of dietary saturated FAs by PUFAs in diet and reverse cholesterol transport. J Lipid Res 2008; 49(11): 2414-8.

[51] Buonacorso V, et al. Macrophage cholesterol efflux elicited by human total plasma and by HDL subfractions is not affected by different types of dietary fatty acids. Am J Clin Nutr 2007; 86(5): 1270-7.

[52] Ku CS, et al. Unsaturated fatty acids repress expression of ATP binding cassette transporter A1 and G1 in RAW 264.7 macrophages. J Nutr Biochem 23(10): 1271-6.

[53] Wang Y, Oram JF. Unsaturated fatty acids inhibit cholesterol efflux from macrophages by increasing degradation of ATPbinding cassette transporter A1. J Biol Chem 2002; 277(7): 5692-7.

[54] $\mathrm{Hu} Y W$, et al. Eicosapentaenoic acid reduces ABCA1 serine phosphorylation and impairs ABCA1-dependent cholestero efflux through cyclic AMP/protein kinase A signaling pathway in THP-1 macrophage-derived foam cells. Atherosclerosis 2009; 204(2): e35-43.

[55] Kammerer I, et al. 13-hydroxy linoleic acid increases expression of the cholesterol transporters ABCA1, ABCG1 and SR-BI and stimulates apoA-I-dependent cholesterol efflux in RAW264.7 macrophages. Lipids Health Dis 10: 222.

[56] Zhang J, et al. Walnut oil increases cholesterol efflux through inhibition of stearoyl CoA desaturase 1 in THP-1 macrophage-derived foam cells. Nutr Metab (Lond) 8: 61.

[57] Zhang $\mathrm{J}$, et al. Alpha-linolenic acid increases cholesterol efflux in macrophage-derived foam cells by decreasing stearoyl CoA desaturase 1 expression: evidence for a farnesoid-X-receptor mechanism of action. J Nutr Biochem 23(4): 400-9.

[58] Kuang $\mathrm{YL}$, et al. Regulation of the expression of key genes involved in $\mathrm{HDL}$ metabolism by unsaturated fatty acids. $\mathrm{Br} \mathrm{J}$ Nutr 108(8): 1351-9.

Received on 16-11-2012

Accepted on 06-12-2012

Published on 31-12-2012

DOI: http://dx.doi.org/10.6000/1929-5634.2012.01.02.1

(C) 2012 Sotherden et al.; Licensee Lifescience Global.

This is an open access article licensed under the terms of the Creative Commons Attribution Non-Commercial License (http://creativecommons.org/licenses/by-nc/3.0/) which permits unrestricted, non-commercial use, distribution and reproduction in any medium, provided the work is properly cited. 